University of New Hampshire

University of New Hampshire Scholars' Repository

Center for Coastal and Ocean Mapping

Center for Coastal and Ocean Mapping

$11-2001$

\title{
GeoZui3D: Data Fusion for Interpreting Oceanographic Data
}

Colin Ware

University of New Hampshire, Durham, colin.ware@unh.edu

Matthew D. Plumlee

University of New Hampshire, Durham, Matthew.Plumlee@unh.edu

Roland J. Arsenault

University of New Hampshire, Durham, Roland.Arsenault@unh.edu

Larry A. Mayer

University of New Hampshire, larry.mayer@unh.edu

Shep M. Smith LT

NOAA

See next page for additional authors

Follow this and additional works at: https://scholars.unh.edu/ccom

Part of the Oceanography and Atmospheric Sciences and Meteorology Commons

\section{Recommended Citation}

C. Ware, M. Plumlee, R. Arsenault, L. A. Mayer, and S. Smith, "GeoZui3D: Data fusion for interpreting oceanographic data," MTS/IEEE Oceans 2001. An Ocean Odyssey. Conference Proceedings (IEEE Cat. No.01CH37295).

This Conference Proceeding is brought to you for free and open access by the Center for Coastal and Ocean Mapping at University of New Hampshire Scholars' Repository. It has been accepted for inclusion in Center for Coastal and Ocean Mapping by an authorized administrator of University of New Hampshire Scholars' Repository. For more information, please contact Scholarly.Communication@unh.edu. 


\section{Authors}

Colin Ware, Matthew D. Plumlee, Roland J. Arsenault, Larry A. Mayer, Shep M. Smith LT, and Donald H. House 


\title{
GeoZui3D: Data Fusion for Interpreting Oceanographic Data
}

\author{
Colin Ware, Matthew Plumlee, Roland Arsenault, Larry A. Mayer, Shep Smith \\ Center for Coastal and Ocean Mapping \\ University of New Hampshire \\ Durham NH 03824 \\ Donald House \\ Texas A\&M University
}

\begin{abstract}
GeoZui3D stands for Geographic Zooming User Interface. It is a new visualization software system designed for interpreting multiple sources of 3D data. The system supports gridded terrain models, triangular meshes, curtain plots, and a number of other display objects. A novel center of workspace interaction method unifies a number of aspects of the interface. It creates a simple viewpoint control method, it helps link multiple views, and is ideal for stereoscopic viewing. GeoZui3D has a number of features to support real-time input. Through a CORBA interface external entities can influence the position and state of objects in the display. Extra windows can be attached to moving objects allowing for their position and data to be monitored. We describe the application of this system for heterogeneous data fusion, for multibeam $Q C$ and for ROV/AUV monitoring.
\end{abstract}

\section{INTRODUCTION}

Many aspects of oceanography are fundamentally multidisciplinary. Seabed properties are influenced by dynamic processes in the water column and vice-versa. Biological activity is related to water column properties, geomorphology and sedimentary processes. In order to better understand these complex ocean interactions we have developed a data visualization tool that supports simultaneous visualization of heterogeneous data sets from a variety of sensor types. We are particularly interested in being able to integrate data from various real-time sensors and fuse them with data already gathered. Here we present for the first time an overview of the system, and some of the applications it has found in oceanography. We also describe some of our future research plans to push the envelope of state of the art $3 D$ interactive visualization.

GeoZui3D has been developed mainly at the University of New Hampshire's newly established Center for Coastal and Ocean Mapping, although it has roots in the Fledermaus visualization system developed originally at the University of New Brunswick $[3,4,8]$ by two of the authors, and others. GeoZui3D, which stands for GeoGraphic Zooming User Interface 3D has three broad goals. First it is intended to push the envelope of interfaces to $3 \mathrm{D}$ geographic information systems (GIS) for visualization and interpretation of multidisciplinary data. Secondly, it is intended to serve as a practical visualization tool for the marine scientists working at the University of New Hampshire as well as partners with whom we collaborate at a number of institutions. Thirdly, we hope to use it as the basis for a tool for public outreach, to help explain aspects of oceanography.

In the remainder of this paper we first describe some of the key features of the user interface and the visualization capabilities of GeoZui3D and then we describe some of the applications, with special emphasis on those that require realtime data input.

\section{$3 D$ User Interface}

GeoZui3D is built first with the user interface in mind, and second with the data in mind. While this may seem a perverse way to design tools for scientists, our experience tells us that the value of tools depends critically on how easy it is to interact with the data and how well the data is represented. Of course the reason we can take this cavalier attitude is that the other issues of data transformation and management are the focus of concern of our excellent collaborators.

Perhaps the most important feature of any 3D visualization system is the ability to rapidly change the viewpoint to better understand the interrelationship of 3D features. GeoZui3D does this with a novel zooming user interface that has the center of the workspace as a key concept. Click on a point of interest and it moves to the center, push the mouse away from you the scene scales up about its center by a factor of approximately 8 times per second, pull it towards you and it scales down at the same rate. Custom widgets illustrated in Fig. 1 enable the data to be rotated around its center or tilted from plan view to horizontal. Interactivity is maintained at all times with an animation governor that measures the time to render the scene during interaction and adjusts the resolution accordingly to get at least ten animation frames per second. Whenever the user stops changing the view the scene is immediately drawn in full resolution. The fact that all interaction is focused around the center of the workspace is important for a number of other aspects of the user interface. It helps link the multiple views when several windows are in use and it is important for stereoscopic display. It also allows us to display the data at higher resolution near the center of the display and this is critical for support of real-time data visualization. 


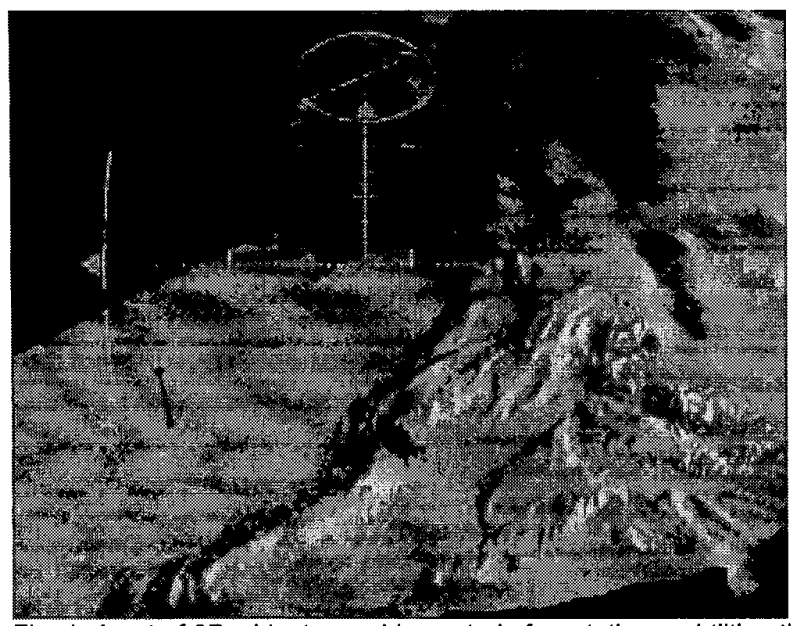

Fig. 1: A set of $3 \mathrm{D}$ widgets provide controls for rotating and tilting the scene about the center of the workspace.

\section{Stereoscopic viewing}

Human stereo vision is optimized for objects at about arms length. If objects are more than about 30 meters away the images are so similar for the two eyes that little stereoscopic benefit is derived. Since "flying" interfaces to GIS data presume a more distant viewpoint they are not suited to stereo viewing, although we have shown previously that artificial manipulations can make stereo viewing usable even while flying over a surface [7]. In GeoZui3D the center of the workspace is geometrically at about arms length which makes stereoscopic viewing very natural without the need for special algorithms. The system supports two kinds of stereoscopic view; either frame sequential, using stereo shutter glasses, or with two orthogonally polarized projectors illuminating an aluminized screen and polarized glasses for viewing. This latter option enables a larger audience to view 3D scenes using low-cost cardboard glasses while the former works well with conventional workstations.

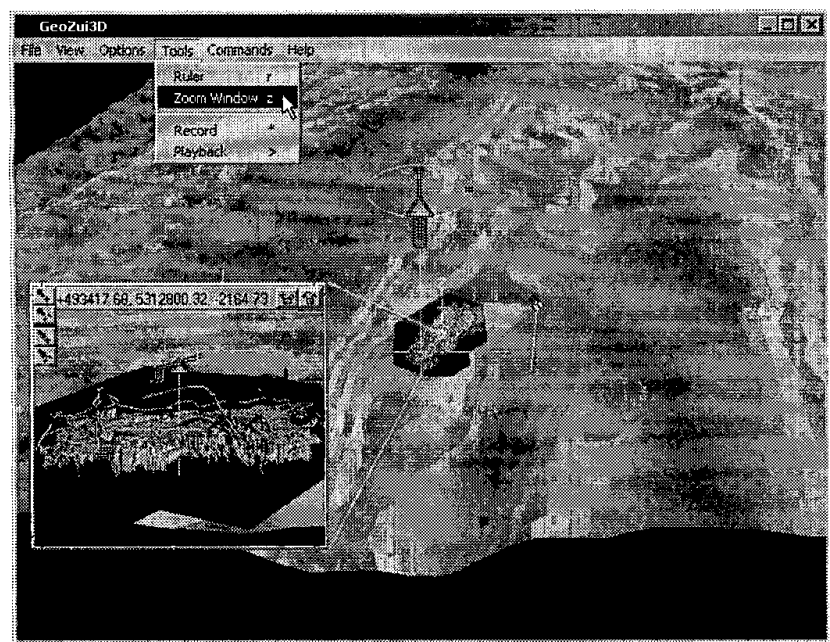

Fig. 2: Windows views can by interactively changed. The source of the window information is shown by the connecting lines, the direction of view is also indicated in the main window.

\section{Multiple Linked views}

A particularly novel feature of GeoZui3D is the way that it supports multiple inter-linked views [5]. It is common for mapping applications to provide an overview window and another magnified window showing part of a map. However, $3 \mathrm{D}$ visualization environments do not typically provide multiple 3D views, at least not linked as they are in GeoZui3D. The advantage of multiple views it that they enable detailed features to be appreciated, together with the broader context in which they exist. In many multi-window systems it can be difficult to understand the relationships between the data in the different views and with $3 \mathrm{D}$ this can be especially problematic. To get around this problem we have implemented Zoomport tethers. Tethers are the lines that connect a zoomport window to the source of its data in the overview as shown in Fig. 2. The tethers lead the eye from the magnified view to what we call a zoomport proxy. This is a representation that shows the virtual camera and its field of view, together with a proxy for the center of the other workspace. Fig. 2 illustrates this feature with a 3D visualization showing the path of the Canadian ROPOS ROV in the broader context of the bathymetry of the region of the Juan de Fuca Ridge Crest where the high resolution data were acquired.

Now we consider how GeoZui3D can be used for oceanographic applications.

\section{SUPPORT FOR MULTIPLE HETEROGENEOUS DATA OBJECTS WITH CONSISTENT GEOREFERENCING}

The basic requirement for data fusion in marine applications is to be able to load multiple different but related objects and view them together. GeoZui3D is an object-oriented system in which data objects can load themselves from a file and display themselves in various ways. Objects can be queried with a mouse click and, through the CORBA interface, can receive and send messages to other entities such as remote sensing systems. This enables GeoZui3D objects to be updated in real-time. GeoZui3D maintains a common georeferencing framework, into which various objects can be drawn so that their inter-relationships can be perceived. To interpret multiple data sources, any number of data objects can be loaded together into this common display space as is illustrated in Fig. 3. Objects can also be hidden or revealed as needed. The following are the major objects currently supported.

- Gridded terrain maps (fusable). Regularly gridded digital terrain maps can be automatically fused with a single command that causes the higher resolution map to drill a holes in lower resolution maps wherever they overlap. This makes it easy to embed high resolution patches of digital map in a lower resolution map. 
- Images. Images can be used in a number of ways in GeoZui3D. Using texture mapping, sidescan sonar or other imagery can be draped on the surface, snapshots can be placed in the 3D model, or a reference grid can be draped. Fig. 4 shows images taken by an ROV placed in situ.

- Tubes. Tubes provide a general purpose tool for displaying Rov tracks, flow lines, or any other linear feature. Tubes can vary in diameter along their length and be color coded to represent variables such as temperature or pressure.

- Curtains. The curtain plot is designed to display continuous water column data or sub-bottom seismic data. An example is Fig. 5 which shows data from a high frequency downward looking sonar designed to measure plankton counts for the GLOBEC project.

- Polygons, lines, polylines, points: Arbitrary 3D structures can be built from these basic primitives.

- Dynamic soundings. The Quad-tree sounding object is specialized for the real-time display of multi-beam soundings.

- Coordinate axes and grids. A variety of methods are implemented for displaying position and scale information. These are adaptive so that they are readable at whatever scale factor is currently being applied.

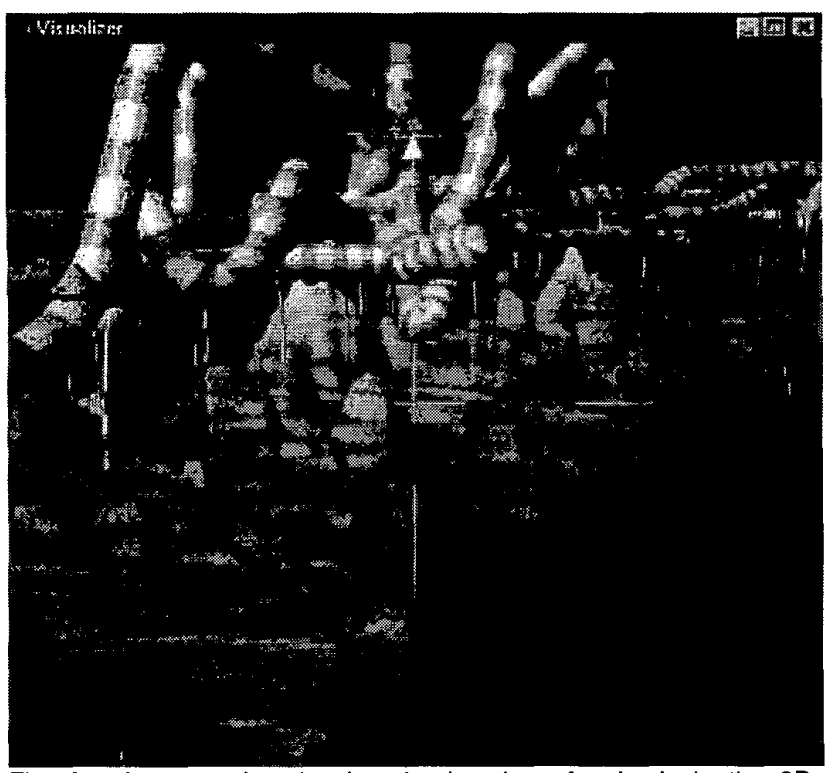

Fig. 3. A composite showing the location of animals in the 3D workspace, as well as the path of the ROPOS ROV over 1 meter resolution bathymetry.

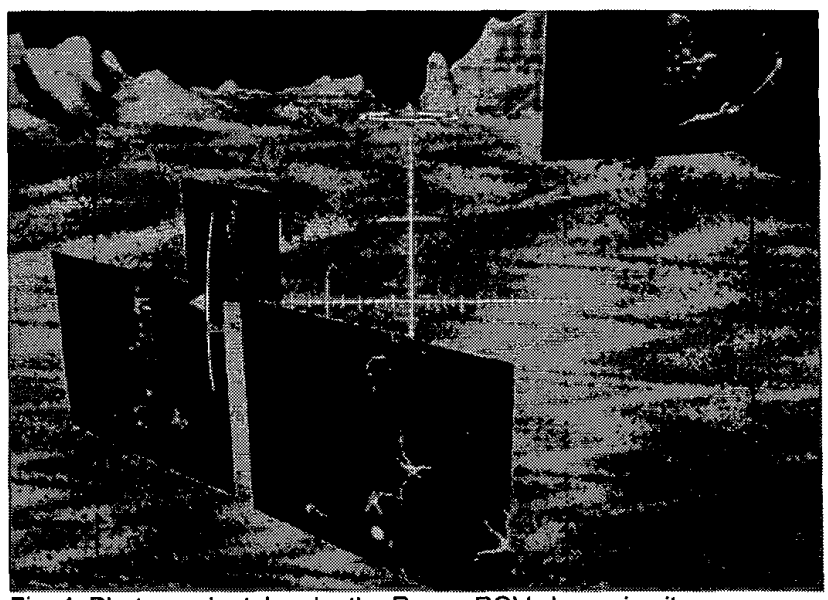

Fig. 4: Photographs taken by the Ropos ROV shown in situ

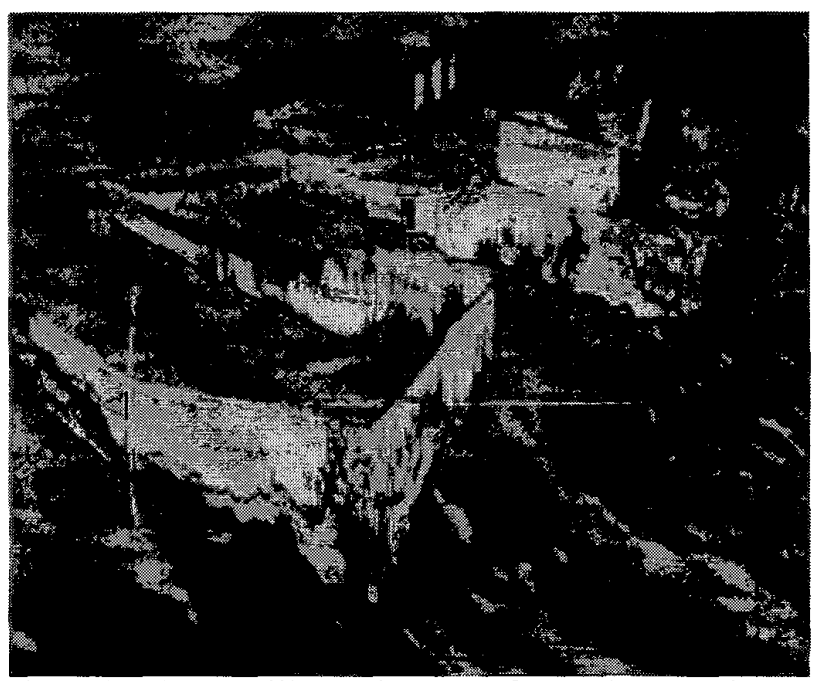

Fig. 5: Curtain Plot: Water column data from a downward looking high-frequency sonar.

\section{$D E M$ Viewer and $Q C$ tool}

GeoZui3D supports the use of draped imagery, allowing charts, sidescan sonar, or any other kind of image to be mapped onto a digital elevation map. GeoZui also supports the placement of non-draped imagery anywhere in a georeferenced space. We have found two uses for this capability. The first is to support QC of multibeam surveys the second is the placement of ROV acquired photographic images in context in a $3 \mathrm{D}$ environment we have already described.

In many survey applications it is common for sidescan surveys to be run in parallel with multibeam bathymetric surveys. Because the sidescan can often show features that are not resolvable in the bathymetric surveys little snips of georeferenced sidescan images are stored to check questionable points in the bathymetry or identify targets. This can often make the difference in determining the validity of the targets. In GeoZui3D we can place the sidescan snippets as horizontal planes offset above the relevant bathymetry. We first load the locations of the snippets into 
GeoZui3D and display them as yellow rectangles above the surface without displaying the images. Hundreds of these can be loaded if necessary. Click once on a rectangle with the mouse and the corresponding images is loaded. Click again and the image become transparent, replaced by a rectangular frame, click again and the imagery reappears. GeoZui3D can load as many as 50 texture images simultaneously. More than this and problems with the graphics card may occur, although this will depend on the specific hardware available. Fig. 6 illustrates this application.

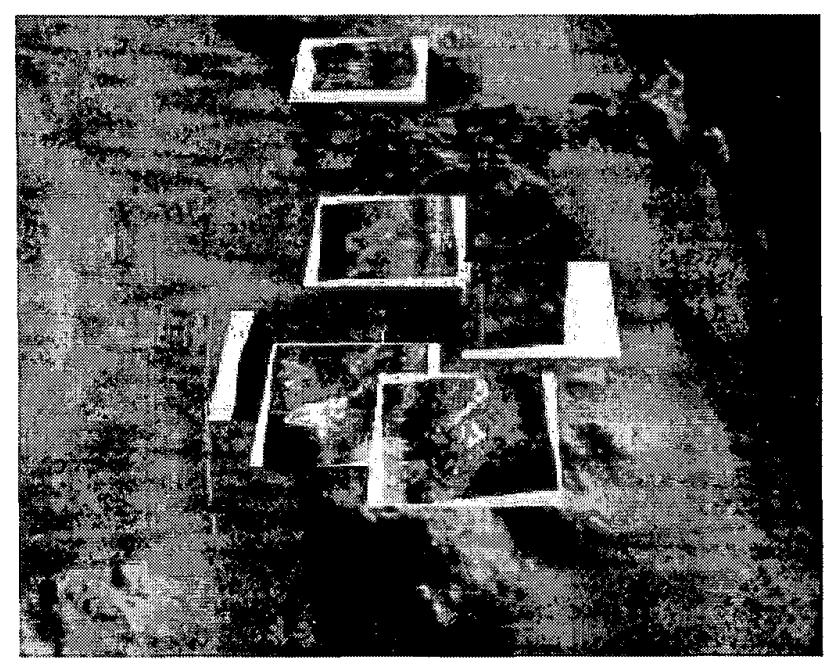

Fig. 6: Sidescan snips displayed above corresponding bathymetry.

\section{SUPPORT FOR REAL-TIME VISUALIZATION}

In the future, ocean mapping is likely to be increasingly done by autonomous or semi-autonomous unmanned vehicles. However, at least in the near-term, even though vehicles are autonomous they are likely to be monitored as closely as is possible, so that if problems do arise they can be easily corrected. Thus for example, C\&C technologies HUGIN AUV continuously returns position information and in addition, the bathymetry measured with the on-board sonar is summarized and transmitted back to the support vessel. We have been working with $\mathrm{C} \& \mathrm{C}$ and with the Autonomous Undersea Systems Institute [2] to understand the requirements for these emerging technologies. To support this and other real-time data input requirements we have created a CORBA interface.

\section{CORBA glue}

CORBA stands for Common Object Request Broker Architecture. It is the leading "middleware" designed to facilitate communications between object oriented software applications. In the case of GeoZui3D, CORBA enables data from remote vehicles, hull mounted sonars, or any other sensor to be linked to the display objects in GeoZui3D. This communication can be bi-directional which allows us to build control interfaces into the system.

\section{Tracking moving objects}

The GeoZui3D user interface supports moving vehicles in a number of ways. When a moving object is being displayed in a window, simply clicking on it changes the focus, so that the center of the workspace becomes locked to the vehicle's position and remains with it as it moves. The direction of view can optionally be changed to be relative to the frame of reference of the vehicle, so that, for example we can choose to always be looking forward "over the shoulder" of the vehicle, or we can view it from the side. Another option is to lock a zoomport proxy to a vehicle. This enables one of the $3 \mathrm{D}$ subwindows to be locked to the moving vehicle, showing its relative position and heading and objects in its vicinity, while the main window provides an overview. The AUSI group is interested in the problems concerning collaborating groups of autonomous vehicles. To support this we can attach zoomport proxies to a number of moving vehicles. Fig. 7 illustrates.

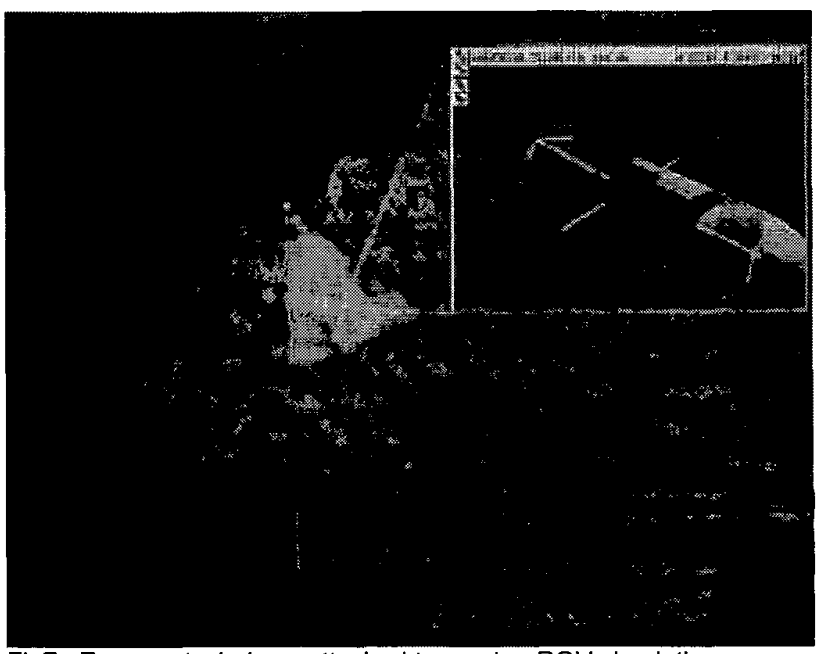

Fig7: Zoomport windows attached to moving ROV simulation.

\section{Quad-trees for real-time monitoring and $Q C$}

It is common practice to use statistical techniques based on "gridding" or "binning" in order to smooth and generate a simplified representation of the large and dense data sets typical of multi-beam echo sounders [6,9]. When this technique is used it is normally necessary to pre-define the gridded area that is of interest. Because of this, binning is normally done after the fact, when the entire survey area has been covered. In order to allow for the real-time application of statistical techniques we have developed an object that incorporates a data structure called a quad-tree. This enables statistical binning techniques to be applied as the survey area grows in arbitrary directions $(1,6)$. This also enables us to display the derived surface in real-time in the context of existing pre-existing data if we have it available. 


\section{CONCLUSION}

GeoZui3D has users at NOAA, CCOM, MBARI and WHOI. The feedback we receive is invaluable in shaping the system. At the same time we are attempting to push the envelope of what an interface to geo-referenced spatial data can be and how users can interact with it. It already incorporates a number of innovations, including the center of workspace interface and the zoomport windows. In addition, we are developing a version that will use a Phantom force feedback device to enable users to feel constraints on a 3D planning task. While it is unlikely that force-feedback will be available for everyday use in the near future, it can be extremely useful in constrained $3 \mathrm{D}$ positioning tasks. In another project we are using a genetic algorithm to develop see-through "lacy" textures enabling one surface to be displayed behind another. This should be useful whenever we wish to interpret features beneath the seabed in the context of the topography of the surface. A third project is designed to exploit a capability of GeoZui3D to record and play back a series of user actions. Using this capability we are working together with the New Hampshire Sea Coast Science Center to create interactive stereoscopic displays that will explain aspects of oceanography to the general public. By adding the ability to record and play back audio segments, we can create self contained 3D tutorials. If and when any of these research capabilities prove themselves to be useful they will be added to the production version of the system and made available to users.

GeoZui3D is freely downloadable from our web site at www.ccom.unh.edu/vislab/GeoZui. The site also contains sample data and a user manual. The manual describes how to use the system for visualization but does not yet document the use of the CORBA interface.

Acknowledgements: A number of individuals have contributed ideas and support, especially Skip Little, Peter Wiebe, and Bob Groman of the Woods Hole Oceanographic Institution. The work is funded by through grants from NSF and NOAA.

\section{REFERENCES}

[1] Arsenault, R, Real time 3D display of hydrographic data for QA and QC. Proc. 2001 U.S. Hydrographic Conference, 2001.

[2] Chappell, S.G. Komerska, R., Peng, L. and Lu, Y. Cooperative AUV Development Concept (CADCON) An Environment for High-Level Multiple AUV Simulation. Proc. $11^{\text {th }}$ International Symposium on Unmanned Untethered Submersibe Technology, Durham NH. 1999.

[3] Mayer, L.A., Dijkstra, S., Hughes Clarke, J.E., Paton, M.and Ware, C., Interactive tools for the exploration and analysis of multibeam and other seafloor acoustic data, in Pace, N.G., Pouliquen, E., Bergem., O., and Lyons., eds., High Frequency Acoustics in Shallow Water, SACLANT
Conf. Proc. Series, CP-45, NATO SACTLANT Research Centre, La Spezia, Italy, p. 355 - 362, 1997.

[4] Paton, M., Mayer, L.A. and Ware, C. Interactive 3-D tools for pipeline route planning. Proc. IEEEOceans'97, Vol. 2 1216-1222. 1997.

[5] Plumlee, M., Komerska, R., Arsenault, R., Ware, C., Chappell, S., and Mayer, L. Monitoring Distributed Autonomous Entities through Linkable 3D Windows. $12^{\text {th }}$ International Symposium on Unmanned Untethered Submersible Technology. UUST01, Proceedings. 2001.

[6] Varma, H.P. Bourdreau, M., McConnel, M. O'Brien, M. and Picott. Probability of detecting error in dense digital bathymetric data sets by using $3 d$ graphics combined with statistical techniques. Lighthouse, 40, 31-36, 1989.

[7] Ware, C., Gobrecht, C., and Paton, Dynamic Adjustment of stereo display parameters. IEEE Trans. on Systems, Man and Cybernetics. 28(1) 56-65, 1988.

[8] Ware, C. and Osborne, S. Explorations and Virtual Camera Control in Virtual Three-Dimensional Environments. Computer Graphics, 24(2) 175-183, 1990.

[9] Ware, C. Slipp, L., Wong, K.W. Nickerson, B., Wells, D. Lee, Y.C. Dodd, D. and Costello, G. A System for Cleaning High Volume Bathymetry. International Hydrographic Review, LXIX(2) Sept. 1992. 